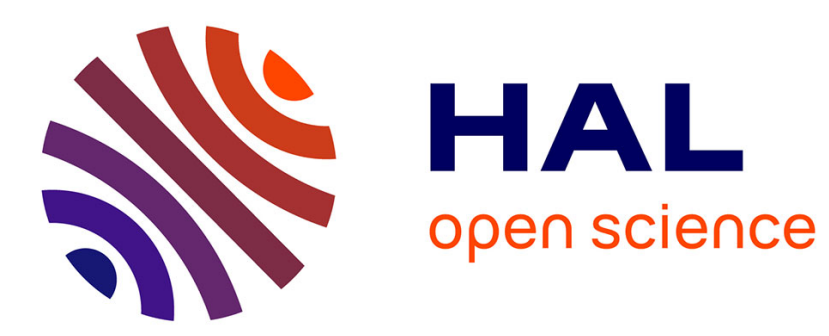

\title{
Resonant amplification of magnetosheath MHD fluctuations at the magnetopause
}

Gerard Belmont, Fabien Reberac, Laurence Rezeau

\section{To cite this version:}

Gerard Belmont, Fabien Reberac, Laurence Rezeau. Resonant amplification of magnetosheath MHD fluctuations at the magnetopause. Geophysical Research Letters, 1995, 22, pp.295-298. 10.1029/94GL03078 . hal-00408543

\section{HAL Id: hal-00408543 https://hal.science/hal-00408543}

Submitted on 4 Feb 2021

HAL is a multi-disciplinary open access archive for the deposit and dissemination of scientific research documents, whether they are published or not. The documents may come from teaching and research institutions in France or abroad, or from public or private research centers.
L'archive ouverte pluridisciplinaire HAL, est destinée au dépôt et à la diffusion de documents scientifiques de niveau recherche, publiés ou non, émanant des établissements d'enseignement et de recherche français ou étrangers, des laboratoires publics ou privés. 


\title{
Resonant amplification of magnetosheath MHD fluctuations at the magnetopause
}

\author{
Gérard Belmont, Fabien Reberac and Laurence Rezeau \\ Centre d'étude des Environmements Terrestre et Planétaires, \\ CNRS/UVSQ, F-78140, Vélizy, France
}

\begin{abstract}
Broad-band ULF fluctuations are routinely observed upstream of the terrestrial magnetosphere: their amplitude, fairly large in the whole magnetosheath, peaks at the magnetopause, and decays to very small values in the magnetosphere. We test the idea that the source of all these fluctuations is in the magnetosheath and that the high magnetopause level is an amplification effect due to propagation. Starting from given stable magnetosonic waves in the magnetosheath, their behaviour is calculated when, during their propagation, they encounter the magnetopause density gradient. For a certain category of incident waves, a "resonant amplificaton", occurring inside the gradient layer, is found.
\end{abstract}

\section{Introduction}

Ultra-Low-Frequency fluctuations are routinely observed at the terrestrial magnetopause with very high intensities [Rezeau et al., 1986, 1989]. These ULF fluctuations are likely to play a very important role in magnetospheric physics because they appear to be the only way to provide transfer of matter (and probably also momentum) through this boundary [LaBelle and Treumann, 1988, Treumann et al., 1992]. Concerning their origin, two different hypotheses can a priori be considered: -1 . the strong fluctuations at the magnetopause are due to local instabilities on the boundary itself or $\mathbf{- 2}$. they are generated in the magnetosheath and they become amplified at the magnetopause. Only the first hypothesis has been addressed in the literature, the various gradients existing at the magnetopause being likely to provide the free energy to many kinds of gradient instabilities; nevertheless, in apparent disagreement with this interpretation, it must be noticed that magnetosheath fluctuations are also regularly observed, with intensity levels significant even if weaker than at the magnetopause [Moustaizis et al., 1986, Engebretson et al., 1991] and with similar spectra [Rezeau et al., 1986]. As it is hard to attribute these fluctuations to magnetopause gradients, it should be accepted, in the framework of the first hypothesis, that two different interpretations, for the magnetosheath and magnetopause fluctuations, coincidently lead to quite similar properties. Moreover, the magnetopause fluctuations themselves, if due to gradient instabilities, should probably show a variability according to the local parameters which is not apparent from the data; (the fluctuations do not disappear when the pressure gradient or the magnetic shear happen to vanish). This paper constitutes the first step for addressing the second hypothesis, i.e. that waves of magnetosheath origin are enhanced at the magnetopause.

In the first section, we recall the main properties of the observed fluctuations that any realistic model should account for. In the following two sections, we present the equations to be solved for describing the propagation of MHD waves through the magnetopause, the simplifications made and the method used for solving. In the fourth section, we give the numerical results concerning the propagation of an incident magnetosonic wave impinging on the magnetopause and describe the "resonant amplification" effect. Finally, we discuss the feasibility of such an interpretation.

\section{Copyright 1995 by the American Geophysical Union.}

Paper number 94GL03078

0094-8534/95/94GL-03078\$03.00

\section{Magnetic fluctuations: observations}

MHD fluctuations at the terrestrial magnetopause have been observed for many years and their properties have been investigated in several papers, in particular those based on data from GEOS 2 and ISEE [Rezeau et $a l ., 1986,1989,1993]$. Figure 1 recalls the main properties of magnetic fluctuations. A temporal sequence of GEOS 2 data is displayed where the spacecraft, because of the magnetopause motion, goes back and forth several times from the magnetosphere to the magnetosheath. Four peaks are evident in the power of the magnetic fluctuations, each of these enhancements (almost one order of magnitude above magnetosheath level) occurring for one magnetopause crossing. Apart from these peaks, it is to be noted that the power in the magnetosheath is significant, still one or two orders of magnitude larger than the magnetospheric level. In summary, it is observed that a non negligible level of fluctuations exists in the magnetosheath, that this level increases drastically at the magnetopause and that it decays to low values on the magnetospheric side. Rezeau et al [1989] emphasized that the fluctuation spectra obey power laws $\mathrm{f}^{-\alpha}$, with $\alpha$ about 3 (the slope being slightly steeper in the magnetosheath than at the magnetopause); this feature, which can be mainly observed above the ion gyrofrequency, is out of the scope of the present paper which deals with an MHD interpretation and thus cannot consistently interpret the phenomena in this frequency range. Concerning the polarization, the conclusion of Rezeau et al [1989] was that the observed fluctuations appear quasi-isotropic in the magnetosheath but predominently perpendicular (to the magnetic field averaged on the period) near the magnetopause.

\section{Propagation of MHD waves through the magnetopause}

To study the propagation of the linear MHD waves through the magnetopause, we assume a slab tangential geometry of the boundary; this means that the waves will be supposed to propagate in a zeroth order medium in which all parameters vary in only one direction, $x$, normal to the magnetopause, and that the zeroth order magnetic field and plasma velocity have no component along the normal direction. In the present paper, we look only for monochromatic solutions; i.e. the first order perturbations $A^{1}$ of all physical parameters $A$ are supposed to vary as

$$
A^{\prime}(x, y, z, t)=A^{1}(x) e^{i\left(k_{r} \cdot \mathbf{r}-\omega t\right)}
$$

(The subscript $T$ labels the "tangential" components, i.e. the projection of the wave vector onto the magnetopause plane and the superscript 1 refers to the first order perturbations of the linearized theory). For these geometrical conditions, which have been widely investigated in the context of Kelvin-Helmholtz studies, the linearized MHD equations reduce to [see for example Walker et al., 1981]:

$$
\begin{aligned}
& \frac{\partial_{x}}{i K_{x}}\left(p_{t}^{1}\right)=G \xi^{1} \\
& \frac{\partial_{x}}{i K_{x}}\left(\xi^{1}\right)=\frac{1}{G} p_{t}^{1}
\end{aligned}
$$

In this notation, $p_{t}^{1}$ is the perturbation of the total pressure $p_{t}=p+B^{2} / 2 \mu_{0} ; \xi^{1}$ is the "normal fluid displacement", derived from the normal velocity by: $\xi^{1}=v_{x}^{1} /(-i \omega) ; K_{x}$ and $G$ are functions of $k_{r}$ and $\omega$ and of the local parameters through the local sound velocity $c_{s}(x)$ and the 


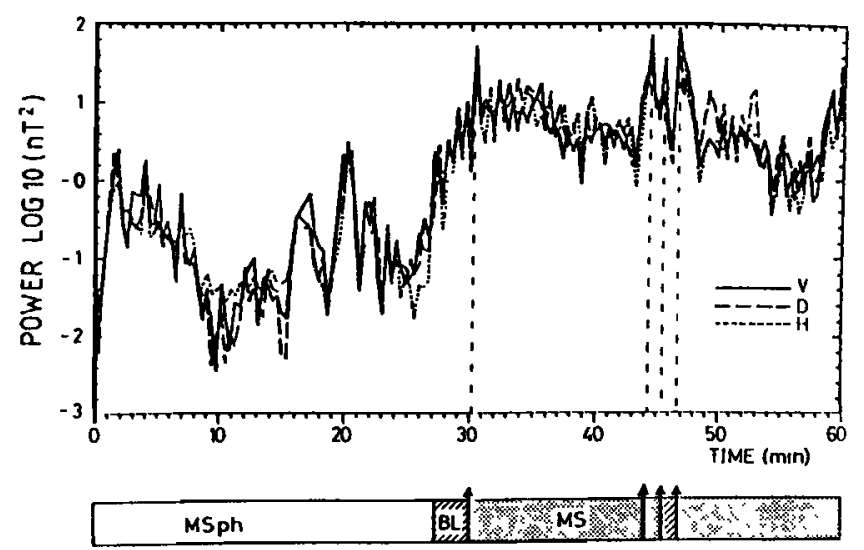

Figure 1. Power of the magnetic fluctuations as observed by GEOS 2. The regions encountered by the spacecraft are indicated at the bottom (magnetosphere, boundary Layer, magnetosheath) and the magnetopause crossings are marked by arrows (the second encounter is partial and the spacecraft goes back and forth in the magnetosheath). The four peaks correspond to the four magnetopause crossings. [After Rezeau et al, 1989].

local Alfven velocity $V_{A}(x)=B_{o}(x) / \sqrt{\mu_{o} \rho(x)}$. They are defined by:

and

$$
K_{x}^{2}=\frac{\omega^{2}}{V_{A}^{2}+c_{s}^{2} \Omega^{2} / \omega^{2}}-k_{T}^{2}
$$

with $\Omega^{2}=\omega^{2}-\left(k \cdot V_{A}\right)^{2}$. Solving for example for $\xi^{1}$, we get the following propagation equation:

$$
\partial_{x}^{2}\left(\xi^{1}\right)+\frac{\partial_{x}\left(G / K_{x}\right)}{G / K_{x}} \partial_{x}\left(\xi^{1}\right)+K_{x}^{2} \quad \xi^{1}=0
$$

For a homogeneous medium, $K_{x}$ and $G$ are constant, and the coefficient of the first derivative $\partial_{x}\left(\xi^{1}\right)$ is zero; then the propagation equation describes the magnetosonic mode, with sinusoidal solutions in $x$ defined by the wave vector $k_{x}= \pm K_{x}$ (which simplifies to $k_{x}= \pm\left[\omega^{2} / v_{A}^{2}-k_{T}^{2}\right]^{1 / 2}$
when $c_{s}=0$ ). When the medium is inhomogeneous, $K_{x}$ and $G$ vary; the coefficient of $\partial_{x}\left(\xi^{1}\right)$ is then no longer zero and this first order term may drastically change the solutions. Since $G=0$ is the dispersion equation of the shear Alfven mode, it can be said that the inhomogeneity introduces a coupling between the magnetosonic mode and the shear Alfven mode. This coupling is especially strong when $G$ happens to be close to zero; if $G=0$ somewhere in the magnetopause gradient, the first order coefficient diverges and the solutions possess a singularity ( $1 / x$ singularity for $\xi^{1}$ if $G$ has a simple zero). We will refer to this phenomenon as "resonant coupling" between the two modes. The mathematical theory of comparable singularities can be found in Sedlacek [1971] for the coupling between electromagnetic ordinary and electrostatic Langmuir modes in a density gradient. The coupling of a fast magnetosonic mode and a shear Alfven mode has been investigated in the frame of fusion studies [e.g. Pritchett and Canobbio, 1981] as well as for space physics, where it has been invoked to interpret the origin of the magnetospheric micropulsations in the PC3-PC5 range [Southwood, 1974, Chen and Hasegawa, 1974, Hasegawa and Uberoi, 1982, Zhu and Kivelson, 1988, 1989]. The role of resonant absorption at thick interfaces has finally been studied by [Hollweg, 1990] for determining the decay of surface modes.

In the following, we will solve the system (1)-(2) for a monochromatic magnetosonic wave launched on the magnetosheath side and propagating earthward (it can be noticed that a shear Alfven wave cannot propagate towards the magnetopause in such a tangential geometry).

\section{Method of solution}

\section{General method:}

In order to solve the propagation equations, it is useful to introduce the variable $\mathrm{g}$ defined by:

$$
g=\frac{p_{t}^{1}}{\xi^{1}}
$$

The propagation equation for $g$ for an incident magnetosonic wave propagating towards the magnetosphere is then a non linear but first order differential equation that we solve numerically:

$$
\partial_{x}(g)=\frac{i K_{x}}{G}\left(G^{2}-g^{2}\right)
$$

The integration is done from right to left $(x=+\infty$ to $x=-\infty)$. The source of the waves being supposed on the magnetosheath side, we know that only one mode can propagate on the magnetospheric side, that is the earthward one: the limit condition at $\mathrm{x}=+\infty$ is thus simply $g=G$. When integrating along decreasing $x, g$ first remains constant (since $G$ itself remains constant far enough from the boundary) and deviates from $G$ in the gradient layer, when $G$ varies. Once the result for $g$ has been obtained, the results for $\xi^{1}$ and $p_{i}^{1}$ can be obtained by the integrations :

and

$$
\begin{aligned}
& \xi^{\prime}=a_{\xi} \exp \int d x \quad i K_{x} g / G \\
& p_{t}^{1}=a_{p} \exp \int d x i K_{x} G / g
\end{aligned}
$$

where $a_{p}$ and $a_{\xi}$ are arbitrary constants characterizing the amplitude of the incident wave. They are related one to the other by $\mathrm{G}=a_{p} j a_{\xi}$ in the quasihomogeneous regions (magnetosheath and magnetosphere).

\section{Simplifications:}

For the sake of simplicity and clarity, and although the equations that are solved are valid in the general case of MHD variations and for any variations of the parameters (in the framework of the tangential geometry adopted), we will restrict ourselfs to the following simplifying assumptions:

- the only zeroth order parameter varying is the density (and consequently the Alfven velocity); this implies that, in this first step, the modulus as well as the direction of the magnetic field are assumed constant througout the resolution domain. The function $G$ can then be put in the simple form: $\Omega^{2}=\omega^{2}-\left(k_{z} V_{A}\right)^{2}$. The density is taken to vary as a hyperbolic tangent across the magnetopause;

- the temperature of the plasma is assumed to be zero $\left(c_{s}=0\right)$; this second simplification allows a clear distinction between the polarizations of the different modes. The function $K_{x}$ is then simply given by:

$$
K_{x}^{2}=\frac{\omega^{2}}{V_{A}^{2}}-k_{T}^{2} .
$$

These assumptions allow simple analytical expressions for all the physical quantities that can be deduced from $\xi^{1}$ and $p_{t}^{1}$. It is easy, for instance, to deduce the different components of the magnetic field perturbation:

$$
\begin{gathered}
B_{x}^{1} / B_{o}=i k_{z} \xi^{1} \\
B_{y}^{1} / B_{o}=-\frac{k_{y} k_{z}}{G} B_{z}^{1} / B_{o} \\
B_{z}^{1} / B_{o}=\frac{p_{1}^{1}}{\rho V_{A}^{2}}
\end{gathered}
$$

\section{Treatment of the singularities:}

In the "resonant case" (i.e. when a point exists in the magnetopause layer where $G=0$ ), there is a singularity in the solutions, causing the numerical algorithm used for solving the differential equation to fail. To remove this difficulty, various tricks can be used. One possibility is to introduce resistivity or viscosity terms into the equations: these terms prevent the perturbations from diverging by bringing some dispersion into play (i.e. higher order differential terms). This method has the disadvantage of being unphysical (there being no binary collisions to justify a resistivity or a viscosity) and to lead to higher order equations, which is cumbersome and obviously unnecessary everywhere except in the very vicinity of the resonance. Another way is to take into account finite Larmor radius corrections [Hasegawa, 1969]; this method is physically realistic but, like the 
preceding, leads to higher order equations. A last method is to consider "global modes" starting from an initial condition and decaying asymptotically with time with complex frequencies ; this method has been used for magnetospheric micropulsations by Zhu and Kivelson [1988, 1989].

We proceed in a different manner, by simply adding a small imaginary part to $k_{z}$ (and consequently another one to $k_{y}$ in order to maintain $\omega$ real); thanks to this artefact, $G$ is then never strictly zero and the singularity is blunted. Physically speaking, this method amounts to assuming incident waves that have amplitudes varying slowly within the initial wave fronts instead of being exactly plane waves. This small departure from planeity, without changing the mathematics, however introduces a coupling between normal and tangential directions that also prevents the perturbations from diverging. The mean (averaged upon the real wavelengths) energy flux, which has diverging $y$ and $z$ components in the purely real case (as $1 / x$ and $1 / x^{2}$ respectively) now has slow variations in $y$ and $z$; since no dissipation is introduced, these variations have to be diverted from the incident $x$ component of the energy flux and, as this incident flux is finite, it can be understood that the $y$ and $z$ components cannot diverge any more.

\section{Monochromatic solutions}

The solutions can be sorted into three different classes (Figure 2) depending on the tangential wave vector $k_{T}$ : the ultimate fate, on the magnetospheric side, of an incident wave is fully determined by the components of $\mathbf{k}_{r}$ compared to the characteristic magnetospheric parameter $\omega / V_{A 2}$ All tangential wave vectors obviously satisfy $\mathrm{k}_{T}<\omega / V_{A l}$ (on the magnetosheath side) since this is the condition for the incident magnetosonic wave to be propagating; inside the circle corresponding to this condition, the three different regions to be considered are:

-class $1: \mathrm{k}_{T}<\omega / V_{A 2}$ : the transmitted mode propagates $\left(k_{x 2}\right.$ real and $\left.k_{x 2}<k_{x 1}\right)$. -class 2: $\mathbf{k}_{T}<\omega / V_{A 2}$ but $k_{T}>\omega / V_{A 2}$ : the transmitted wave is evanescent $\left(k_{x 2}\right.$ imaginary), without resonant effect.

-class 3: $\mathbf{k}_{T}>\omega V V_{A 2}$; the transmitted wave is again evanescent, but a resonant effect occurs; (one point exists in the boundary where $V_{A}=\omega / k_{z}$, i.e. $G=0$ ).

In the case of an infinitely thin boundary, a complete study of reflection and refraction of MHD waves at the magnetopause can be found in Verzariu [1973]. The results presented hereafter have been obtained with the following parameters: magnetopause thickness $a=600 \mathrm{~km}$; Alfven velocities $V_{A 1}=200 \mathrm{~km} . \mathrm{s}^{-1}$ in the magnetosheath, $V_{A 2}=600 \mathrm{~km} . \mathrm{s}^{-1}$ in the magnetosphere; densities $n_{1}=50 \mathrm{~cm}^{-3}, n_{2}=5 \mathrm{~cm}^{-3}$; frequency $f=0.5 \mathrm{~Hz}$.

Figure 3 displays the modulus of the magnetic field vector perturbation as calculated for one wave of each category. For observing the spatial variations across the boundary of this modulus, the extreme values, min and $\max$, of $\left|\mathbf{B}^{1}\right|(\mathrm{t})$ are displayed; they are calculated from the formula:

$$
\left|\mathbf{B}^{1}\right|_{\text {exx }}^{2}=\frac{1}{2}\left(\left|B_{x}^{1}\left\|^{2}+\left|B_{y}^{1}\right|^{2}+\right\| B_{z}^{1} \|^{2} \pm\right|\left(B_{x}^{1}\right)^{2}+\left(B_{y}^{1}\right)^{2}+\left(B_{z}^{1}\right)^{2} \mid\right)
$$

(The double bars on the RHS represent the modulus of complex numbers while the simple bars on the LHS represent the modulus of a vector). The modulation observed on the magnetosheath side corresponds to structures

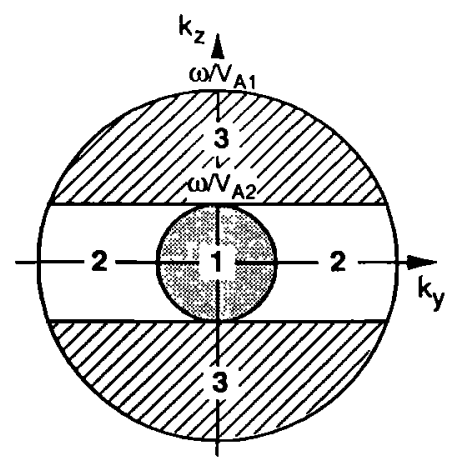

Figure 2. Projection of the wave vector onto the magnetopause plane and subsequent classification of the incident waves (see text).
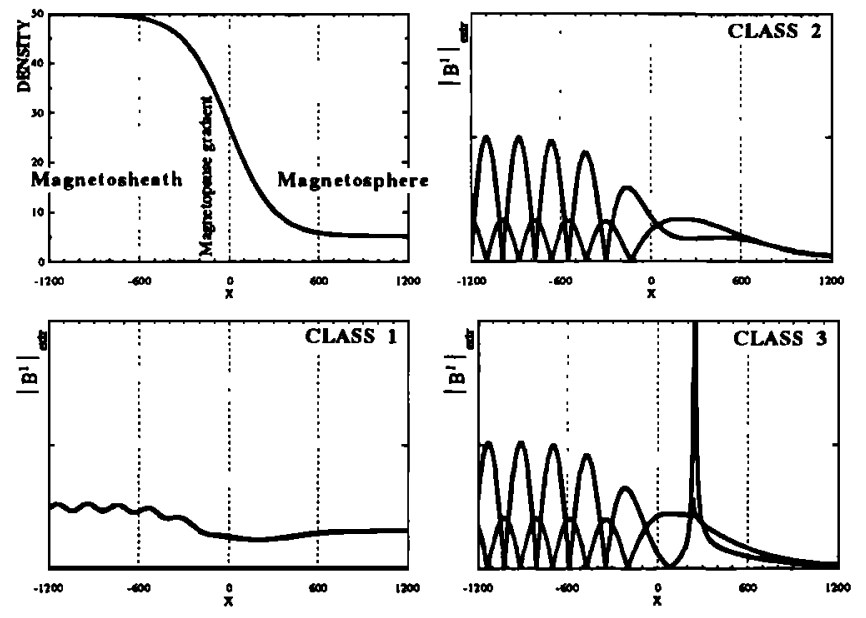

Figure 3. Spatial variations across the boundary of the modulus $\left|\mathbf{B}^{1}\right|$ of the magnetic field vector perturbation for the three classes of incident waves. (The extreme values, min and max, of $\left|B^{1}\right|(t)$ are displayed). The $x$ axis is the magnetopause normal. The magnetopause gradient lies approximately between the abscissas -300 and $+300 \mathrm{~km}$; the first panel displays the given density variation for reference.

of standing waves due to reflection; this reflection is total for class-2 and -3 waves and partial for a class- 1 wave. The first interesting point to be noticed is the absence, with the parameters used, of a large scale regular amplification (of the type of an Airy function) that is usually obtained in the vicinity of any reflection point for a customary propagation equation (without a first order term). Instead of that, the most striking feature of these results is the occurrence in one case (class 3 ) of a very high and narrow peak which is the mark of the Alfven resonance. We call this phenomenon of a very localized enhancement of some components of the incident wave "resonant amplification". For the same class 3 wave, the three components and the modulus of the magnetic perturbation are also displayed for intercomparison in Figure 4. It is observed that the $B_{2}^{1}$ component (parallel to the static magnetic field) does not diverge, while the $B_{x}^{1}$ and $B_{y}^{1}$ components diverge as $\ln (x)$ and $1 / x$ respectively. This can be easily demonstrated analytically from the propagation equation. These results are very analogous to those of $Z h u$ and Kivelson [1988]. The strongest divergence (the $B_{y}^{1}$ one) determines the behaviour of the modulus in this region. It is worth noticing that, if the shapes displayed for the divergences are accurately determined, the higher points represented cannot be regarded as meaningful and representative of realistic maximum values. It must be kept in mind that, for an exact plane wave, these maximum values should be infinite; thanks to the imaginary parts introduced for $k_{z}$ and $k_{y}$, they are limited to finite (and high) values, but these finite value themselves cannot be determined from such displays because the peaks happen to be sharper than the mesh used for integration and visualization. This point could represent a difficulty if we wanted to make a quantitative comparison between the model results and the experimental data; we will show in the following section that, at the present stage of the work, such comparisons are however out of reach for more fundamental reasons.

\section{Discussion and conclusion}

The fluctuations observed at the magnetopause are generally supposed to be locally generated, the possibility of a convection from the magnetosheath being rarely suggested (except for a remark concerning mirror modes in the conclusion of Treumann et al., 1994). Is it possible to interpret the high level of fluctuations at the magnetopause as the effect of a resonant amplification of the magnetosheath fluctuations? This paper constitutes a first attempt to answer this question. Having investigated the propagation of sinusoidal monochromatic magnetosonic waves through the magnetopause layer under some restrictive conditions, several factors 

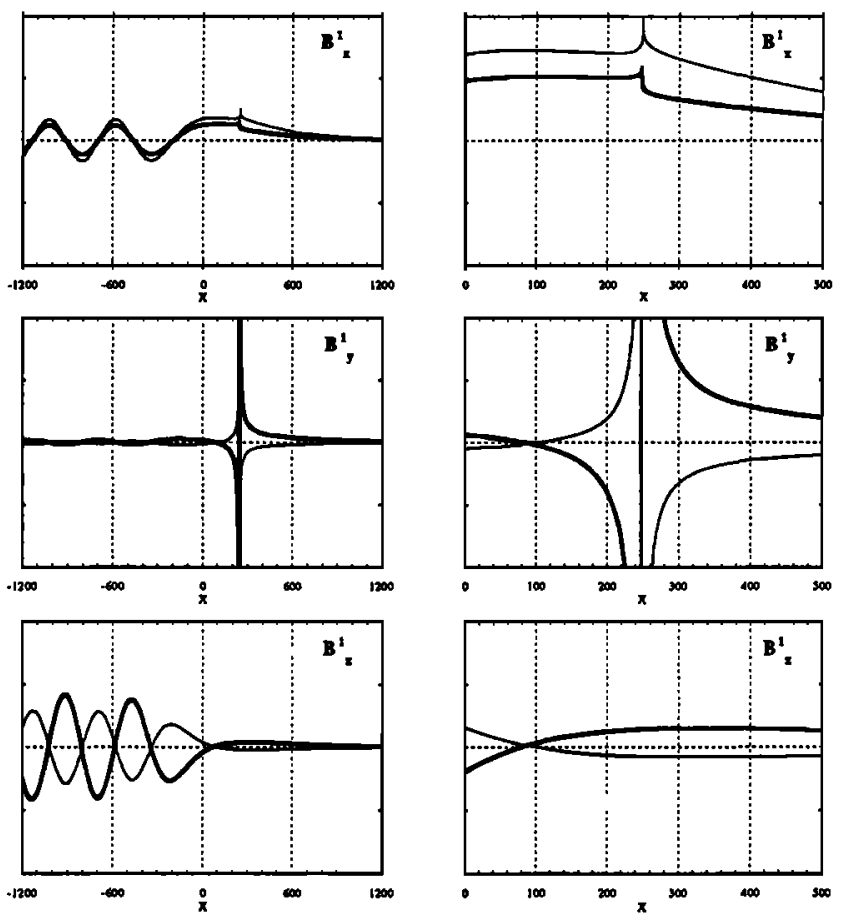

Figure 4. Perturbation of the three magnetic field components for a wave of class 3 (thick line=real part, thin line=imaginary part). The zone of the singularity is enlarged in RHS column.

have been brought up that will help to decide. Comparing the experimental evidence to the model predictions, the following points can be outlined: -Fluctuation level in the magnetosphere: it is observed to be much weaker than in the magnetosheath. This is quite consistent with the fact that -i) most of the possible incident waves are reflected at the magnetopause (cf. Figure 2: region 1 has a surface much smaller than regions 2 and 3) and -ii) the few transmitted waves that are propagating into the magnetosphere often have amplitudes smaller than in the magnetosheath. The amplitude variation can be estimated analytically by invoking the conservation of the mean energy flux in the $x$ direction: for the case, for example, of a quasinormal incident wave with $k_{x} \gg k_{y}$, it can be shown that the main component $B_{2}^{1}$ varies as $\sqrt{G}$, i.e. decreases with $x$ approximately as $\sqrt[4]{\rho}$.

-Fluctuation level at the magnetopause: higher than the magnetosheath level. This is consistent with the fact that some components of the magnetic perturbation diverge in the gradient layer, leading to very high values in very narrow localized peaks. This phenomenon occurs for all waves of class 3, which a priori is the most common category in Figure 2. Nevertheless, a quantitative comparison is not possible at this stage, firstly because of the numerical difficulties mentioned in the preceding section concerning the levels reached at resonance and, more fundamentally, because the spectrum of fluctuations in the medium is continuous and the extrapolation to this case from monochromatic results is not straightforward. The resonance peak of each monochromatic component will occur at different locations inside the layer (depending on $\omega / k_{z}$ ); all these peaks will superimpose in a complex way, giving a final level and spatial shape which have not yet been calculated. It is to be noticed that the problem of a continuous spectrum has never been investigated in the literature. The initial value approach [Zhu and Kivelson, 1988, 1989, Hollweg, 1990], even if leading to a slight widening in $\omega$ because of the decay of the global modes, has only been applied, up to now, to mono- $k_{z}$ waves (or to a superposition of a few such waves); moreover, this approach does not seem appropriate to model a problem where the energy source is localized in space (incident waves on one side) and not in time.

-Polarization: observed preferentially perpendicular to the static magnetic field. Again, this is consistent with the calculation because the diver- gences have been shown to appear only in the two perpendicular components, while $B_{2}^{1}$ is not divergent. Nevertheless, a more quantitative comparison between the levels of the three components is out of reach of the present calculation which does not account for the existence of continuous spectra. It would also require to drop the simplifying assumptions (cold plasma, constant magnetic field) which may alter the polarizations noticeably.

Though some important questions cannot be answered by the present simple treatment, we can in conclusion state that the results obtained compare quite well with the experimental data. We therefore believe that resonant amplification of MHD waves at the magnetopause is an important process for its dynamics.

Acknowledgments. We are pleased to thank M. Kivelson for fruitful discussions and suggestions. We are also indebted to the referee $R$. Treumann for many linguistic improvements of the paper.

\section{References}

Chen, L., and A. Hasegawa, A theory of long period magnetic pulsations, 1. Steady state excitation of field line resonance, J. Geophys. Res., 79, 7, 1024-1032, 1974. Engebretson, M.J., N. Lin, W. Baumjohann, H. Luehr, B.J. Anderson, L.J. Zanett1, T.A. Potemra, R.L. McPherron, and M.G. Kivelson, A comparison of ULF fluctuations in the solar wind, magnetosheath, and dayside magnetosphere, 1. Magnetosheath morphology, J. Geophys. Res., 96, A3, 3441-3454, 1991.

Hasegawa, A., and C. Uberoi, The Alfven wave, Technical Information Center, U.S. Dept. of Energy, Washington D.C., 1982.

Hollweg, J.V., Resonant decay of global MHD modes at thick interfaces, $J$. Geophys. Res., 95, A3, 2319-2324, 1990.

Labelle, J.,and R.A. Treumann, Plasma waves at the dayside magnetopause, Space Sci. Rev, 47, 175-202, 1988.

Moustaizis, S., D. Hubert, A. Mangeney, C.C. Harvey, and C. Perche, Magnetohydrodynamic turbulence in the earth magnetosheath, Ann. Geophys., 4, A5, 355-362, 1986.

Pritchett, P.L., and E. Canobbio, Resonant absorption of Alfven waves, Phys. Fluids, 25, 2374, 1981.

Rezeau, L., S. Perraut, and A. Roux, Electromagnetic fluctuations in the vicinity of the magnetopause, Geophys. Res. Lett., 13, 1093-1096, 1986.

Rezeau, L., A. Morane, S. Perraut, A. Roux and R. Schmidt, Characterization of Alfvenic turbulence in the magnetopause boundary layer, J. Geophys. Res., 94, 101-110, 1989.

Rezeau, L., A. Roux, and C.T. Russell, Characterization of small scale structures at the magnetopause from ISEE measurements, J. Geophys. Res., 98, A1, 179-186, 1993.

Sedlacek, Z., Electrostatic oscillations in cold inhomogeneous plasma, 1. Differential equation approach, J. Plasma Phys. 5, 239, 1971.

Southwood, D.J., Some features of field line resonances in the magnetosphere, Planet. Space Sci., 22, 483-491, 1974.

Treumann, R.A., J. LaBelle, and R. Pottelette, Plasma transport through magnetic boundaries, in Proceedings of the 26th ESLAB Symposium, Study of the SolarTerrestrial system, held in Killarney, Ireland, 16-19 June 1992, ESA SP-346, 1992.

Treumann, R.A., J. LaBelle, and T.M. Bauer, Diffusion processes: an observational perspective, in Physics of the magnetopause, edited by P. Song and B.U.O. Sonnerup, AGU monographs, in press, 1994.

Verzariu, P., Reflexion and refraction of hydromagnetic waves at the magnetopause, Planet. Space Sci., 21, 2213, 1973.

Walker, A.D.M., The Kelvin-Helmholtz instability in the low latitude boundary layer, Planet. Space Sci., 29, 1119, 1981.

Zhu, X, and M. G. Kivelson, Analytic formulation and quantitative solutions of the coupled ULF wave problem, J. Geophys. Res., 93,A8, 8602-8612, 1988.

Zhu, X, and M. G. Kivelson, Global mode ULF pulsations in a magnetosphere with a non monotonic Alfven velocity profile, J. Geophys. Res., 94,A2, 1479-1485, 1989.

G. Belmont, F. Reberac and L. Rezeau, CETP, 10-12 Avenue de l'Europe, F78140, France (e-mail: Gerard.Belmont@ cetp.ipsl.fr)

(Received Sepetember 27, 1994; accepted november 4, 1994) 